\title{
STUDY ON THE LINE SCAN CCD CAMERA CALIBRATION OF VEHICLE-BORNE 3D DATA ACQUISITION SYSTEM
}

\author{
Youmei Han ${ }^{\mathrm{a}, \mathrm{b}^{*}}$, Bogang Yang ${ }^{\mathrm{b}}$, Feizhou Zhang ${ }^{\mathrm{a}}$ \\ a. School of earth and space sciences, Peking University, Beijing, China, E-mail: \\ youmei.han@gmail.com(Corresponding)
}

b. Beijing Institute of Surveying and mapping, Beijing, China

V/5: Image Sensor Technology

KEY WORDS: Line Scan Camera; Vehicle-borne; dynamic calibration; Laser Scanner; Vehicle-borne

\begin{abstract}
:
Based on the characters of the line scan CCD camera and the Vehicle-borne 3D data acquisition system, it presented a novel method to calibrate the line Scan Camera (LSC) based on the laser scanner. Using the angle information in the original laser scanner data, combing the principle of the line scan camera, it built a calibration model for LSC and designed some experiment methods to implement that. Using the new model and the special experiment methods it computed out high precision LSC calibration parameters, which provides basis for the fusion of image and point cloud data and gives references to the similar sensors calibration.
\end{abstract}

\section{INTRODUCTION}

Building surface texture data acquisition and real texture mapping has been a difficult problem which City Virtual Reality and Visualization should solve, and it is also one key problem of the 3D city modeling (WEI Bo and ZHANG Aiwu, 2009). Vehicle-borne 3D data acquisition system (Figure 1) is a good system for City Virtual Reality and Visualization, which has accelerated the pace of urban 3D modeling (CHEN Yunfang and YE Zetian, 2006). Meanwhile in this 3D data acquisition system, using line scan camera to capture the true texture makes the city modeling more real.

Vehicle-borne 3D data acquisition system consists of line scan camera, laser scanner, GPS, Inertial Measurement Unit (IMU) etc. Line Scan Camera (LSC) has many advantages in texture capturing, such as high frequency, wide angle, real color etc.. It can save the data in time and doesn't lead to image missing which the planar camera couldn't reach. While its' non-metric and lens distortion are still the key problems to be used as a main sensor to capture the texture data. Traditional planar camera calibration methods, such as spatial resection, direct linear transformation, Vanishing Point method based on multi images, analytical plumb line, as well as self-test method (XIE Wenhan and ZHANG Zuxun, 2004; FENG Wenhao, 2004; LIN Zongjian and CUI Hongxia, 2005; Wang Dong etc. 2007), which always rely on the different images that captured on different direction then calculate the internal elements and their lens distortion based on co-linearity equation. Now regarding the line scan camera as a special case of the planar camera, its' special features need explore new methods to calibrate it. Until now, most of the calibration methods for the line scan camera are to fix the camera position and move the targets to get the calibration data (Radu Horaud etc., 1993; QU Wenqian etc., 2008; ZHANGHongtao and DUAN Fajie, 2007), which have low precision because of the short distance between the camera and the targets.

* Corresponding author: Youmei Han, email: youmei.han @.gmail.com, tel: +8613426280276
Line Scan Camera (LSC) and Laser Scanner are the two important components of the Vehicle-borne 3D data acquisition system. The former is used to obtain the building color texture information on both sides of the street and the latter can obtain the corresponding high-precision three-dimensional coordinates of the building. They have a common feature: the laser scanner data can be seen as lines composed of discrete points, and line scan camera data are RGB color lines with one pixel width. So this paper presented a novel approach which is different from traditional methods of planar camera calibration and current methods of the line scan camera calibration and can provide reference for similar linear array CCD sensors calibration.

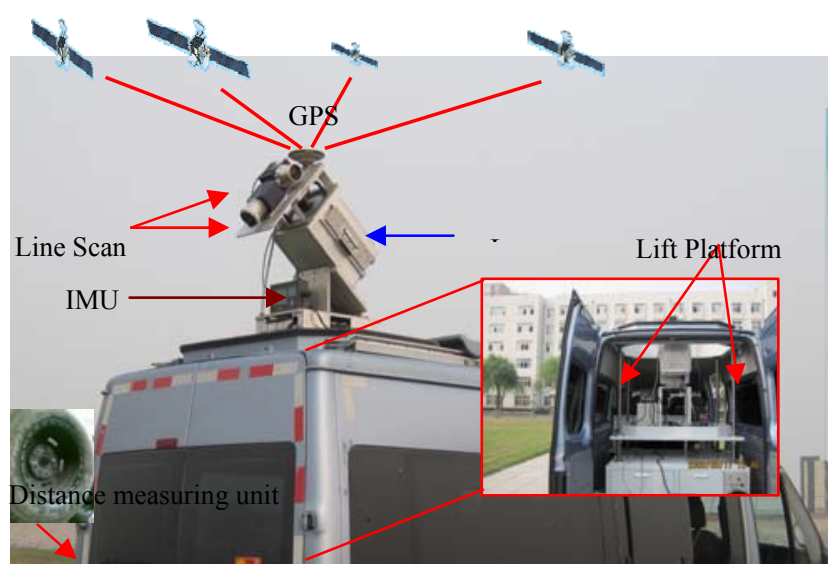

Figure1 The Vehicle-borne 3D data acquisition system (Made by China Capital Normal University) 


\section{THE CALIBRATION PARAMETERS OF THE LSC}

The line scan camera XIIMUS CL made by Finland TVI Company is used by Vehicle-borne 3D data acquisition system, which has been integrated into the Vehicle-borne 3D data acquisition system. This camera uses the prism spectroscopic technique, which has good color reproduction. The white balance function button is on the back of the camera which is very convenient and efficient. Its frequency can reach to $9.5 \mathrm{k}$ lines per seconds. It also has high resolution with $10 \mu \mathrm{m}$ pixel size which can get very real colorful image. However, it should be calibrated to eliminate mechanical error, and then the image data can be fused with the point clouds to get good information for the City Virtual Reality and Visualization.

The errors of the LSC mainly come from the lens distortion and the CCD structure itself. This LSC uses a Nikon AF lens (the focus length is $24 \mathrm{~mm}$ ). The error caused by the lens comes from lens design, production and assembly which lead to image pixel deviate from its correct location. It is also named as the optical distortion, which includes radial distortion and eccentric radial distortion. Eccentric distortion is always less than one-third of one pixel, so here it only discusses the radial distortion (WANG Liuzhao, 2006).

The CCD errors of LSC always come from CCD placement, unevenness of CCD planar and the distortion of each CCD unit. The CCD placement can result in the distortion of the principal point; the unevenness of CCD planar, which can only be calibrated by special device directly, has nothing to do with photography. In fact it is too small to influence the whole LSC calibration (XIE Wenhan, ZHANG Zuxun, 2004).

\section{THE IMPLEMENTATION OF THE CALIBRATION METHOD FOR THE LSC}

\subsection{Discuss of the existing methods}

Line Scan camera should move around the target objects then the normal images of the objects can be grabbed. However, when it is moving the position and the relative attitude is changing over time. If it uses the traditional plane-array camera calibration methods to calibrate LSCs, the relative attitude could not be saved because of the high frequency of the camera shutter itself, which is the key problem to get the orientation elements by traditional methods.

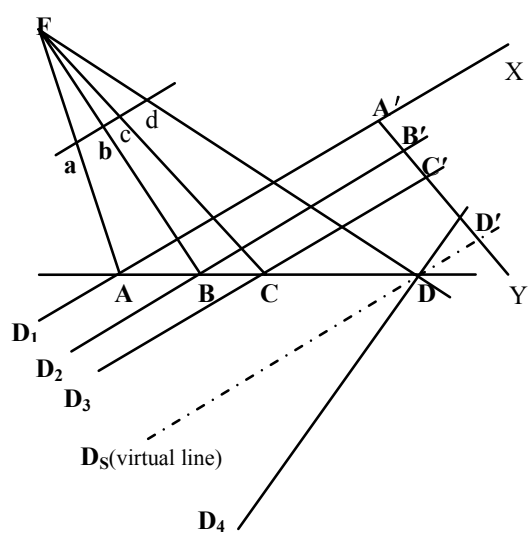

Figure 2 The special targets from R. Horaud (1993)

Until now many experts have provided some methods, such as: R. Horaud designed a special graph for the LSCs in 1993, which was made of several straight lines (Figure 2), some scholars comes from Capital Normal University(China), have also done some similar experiments (QU Wenqian etc., 2008, Figure 3).

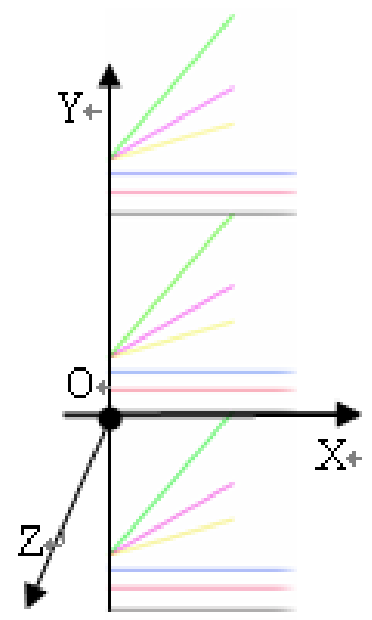

Figure 3 The special target designed by QU Wenqian (2008)

In 2008, Beijing Information Science and Technology University invented a LSCs calibration target which also was made by a number of equally spaced vertical and horizontal lines. Tianjin University (China) has proposed a two-steps method to calibrate line CCD, which is also based on this similar design principle. Their common feature is that based on this kind of specific targets, analyzed the images of the special lines on the targets to get the lines' relative positions relationship, then using this data to calibrate LSCs' elements of interior orientation. If the target was far from the camera, the image of it would be very small on the whole image. In order to get the target image on the edge of the frame, very big target should be designed which is impossible and not convenient to do this. So the accurate of these kinds of methods is relatively low.

3.2 The idea of the novel calibration method based on the laser scanner

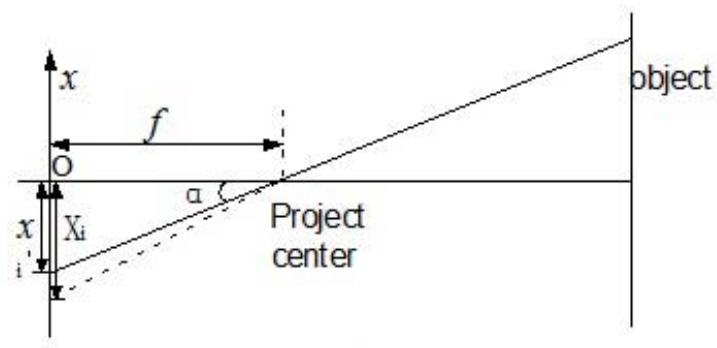

Figure 4 The Imaging Principle of the Line Scane Camera

In Figure 4, regarding the middle of the line image (named as $\mathrm{O}$ ) as the origin, with the positive direction pointing to the top of the camera, one image plane coordinate system was built (Figure 4). In this paper, the main calibration content is camera inner orientation elements, which mainly includes the projection of the principal point on the image coordinate system $\left(\mathrm{x}_{0}\right)$, the camera focal length $\mathrm{f}$ and radial distortion. From Figure 4, it can conclude that:

$$
x_{i}=\tan \alpha_{i}^{*} f
$$


Where: $X_{i}$ is the theory value of the object on the image coordinate system, $\mathrm{f}$ is the focal length, $\alpha_{i}$ is an angle between the line from some pixel (here name it as $\mathrm{Z}$ ) to the principle point and the axis (it is also called incident angle).

Above all, the real image coordinate of the objects only has to do with incident angle and the focal length. As long as these two parameters could be determined accurately, it also could get the real image coordinate then get interior orientation elements mentioned of the LSC accurately.

In the Vehicle-borne 3D data acquisition system, the laser scanner captures the 3D coordinate data in high frequency and it has relatively high angle measure unit. The nominal accuracy of the laser scanner is \pm 0.6 ' (Wang Liuzhao and $\mathrm{Qu}$ Laichao, 2009).The normal focal length of the XIIMUS CL lens is $24 \mathrm{~mm}$ ( each pixel size is $10 \mu \mathrm{m}$ ).

Formula (1), $x_{i}=\tan \alpha_{i}^{*} f$, when $\alpha_{i}$ is a small value, it can be written as $x_{i}=\alpha_{i}^{*} f$

$$
M_{x}=f * m_{a}= \pm 0.42 \text { (Pixel) }
$$

Where $m_{a}$ is $\pm 0.6^{\prime}$, and $\mathrm{f}$ is $24 \mathrm{~mm}$. That is to say the influence on the image coordinate comes from the angle measure is less than 0.5 pixel size. So the angle distortion can be ignored during the calibration of the LSC. The LSC was fixed on the lasers scanner by precision machine (Figure 5), and the installation error can be controlled within $\pm 1 \mathrm{~cm}$. When the distance between the objects and the camera is longer than $25 \mathrm{~m}$, according to formula (1), it can get the eccentric error of these two sensors, which is about 0.4 pixel sizes (each pixel size is 10 $\mu \mathrm{m})$. So this small error could be ignore, which is a very important primary condition to propose this novel calibration approach.

Fixed the LSC on the top of the laser scanner firmly, and made the working faces of these two sensors closed to parallel which can be regarded as a whole rigid body. Meanwhile the intersecting angle was adjusted within \pm 18 " by precision feeler gauge. This rigid parallel structure makes they keep the same attitudes during moving.

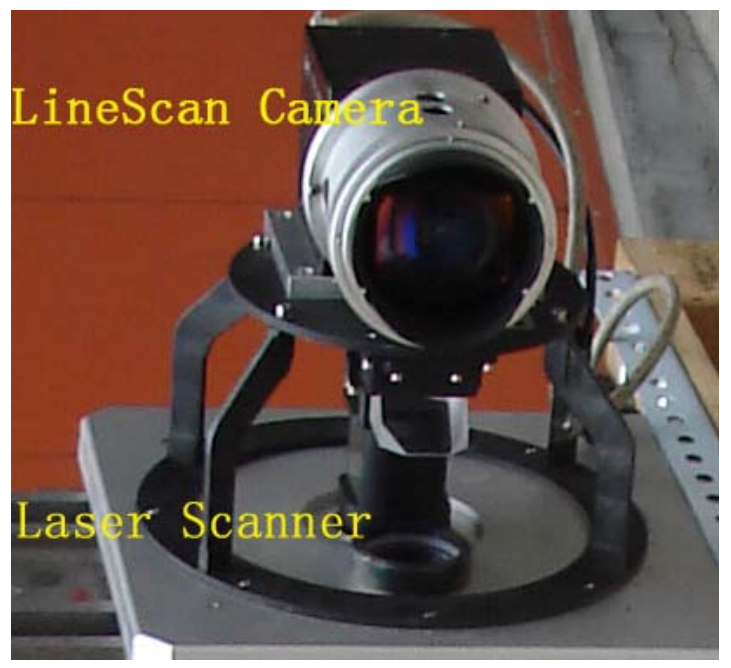

Figure 5 The rigid construction of the LSC and LS

Moved this rigid body along a precision guide rail, then it could get the images and the geometrical information (cloud points) of the objects. It used a building wall as the object which has much special characteristic information. Extract characteristic points and the corresponding incident angle of them from the cloud points by programming. Obtain the corresponding points' pixel from the line scan images and make it group with the cloud points extracted above. It needed to observe some groups of data which distribute on the wall evenly. Then it used these two kinds of information as the original data to compute out the LSC parameters by precision adjustment computations. That is the main idea of this novel approach.

\subsection{The calibrate model of this approach}

According to the geometrical optics, the LSC' $y=0$, the radial distortion of its lens $\Delta r$ can be written as a homogeneous equation as following:

$$
\Delta_{r}=x\left(k_{0} r^{2}+k_{1} r^{4}+k_{2} r^{6}+\ldots\right)
$$

Where $\Delta r$ ' unit is $\mu \mathrm{m}, k_{i}(i=0,1,2, \ldots$.$) is the coefficient$ to express the lens of the camera, $r$ is the radius vector of some pixel. The $\Delta r$ is very small, so it can be written as:

$$
r=\sqrt{\left(X^{\prime}-x_{0}\right)^{2}}
$$

Where $X_{0}$ and $X^{\prime}$ are the image coordinate of the principal point and this point on the image.

For most of the lens, three $k$ coefficients are enough to describe the deviation curve. However two is enough for some good lens (FENG Wenhao, 2004).

Using formulas (1), (2) and (3), it constructed the calibrate model of the LSC:

$$
\begin{gathered}
\tan \alpha_{i}{ }^{*} f=\left(X_{i}^{\prime}-x_{0}\right)+k_{0}\left(X_{i}^{\prime}-x_{0}\right)^{3} \\
+k_{1}\left(X_{i}^{\prime}-x_{0}\right)^{5}+k_{2}\left(X_{i}^{\prime}-x_{0}\right)^{7}
\end{gathered}
$$

Because of $f$ is related with $x_{0}, k_{0}, k_{1}, k_{2}$, it needs to compute these parameters by two steps in order to get high precision results.

Firstly, compute $x_{0}, k_{0}, k_{1}, k_{2}$

Carrying on the linearization to this model it can get formula (4) as following,

$$
\begin{aligned}
v_{x_{i}}= & \left(x_{i}\right)-x_{i}+\left(1+3 k_{0}\left(X_{i}^{\prime}-x_{0}\right)^{2}+5 k_{1}\left(X_{i}^{\prime}-\right.\right. \\
& \left.\left.x_{0}\right)^{4}+7 k_{2}\left(X_{i}^{\prime}-x_{0}\right)^{6}\right) \Delta x_{0}+\left(X_{i}^{\prime}-x_{0}\right)^{3} \\
& \Delta k_{0}+\left(X_{i}^{\prime}-x_{0}\right)^{5} \Delta k_{1}+\left(X_{i}^{\prime}-x_{0}\right)^{7} \Delta k_{2}
\end{aligned}
$$

With a lot of observation value, formula (4) can be written as a matrix:

$$
V=A X-L
$$

here:

$$
\begin{aligned}
& A=\left[\left(X_{i}^{\prime}-x_{0}\right)^{3},\left(X_{i}^{\prime}-x_{0}\right)^{5},\left(X_{i}^{\prime}-x_{0}\right)^{7},\right. \\
& \left.1+3 k_{0}\left(X_{i}^{\prime}-x_{0}\right)^{2}+5 k_{1}\left(X_{i}^{\prime}-x_{0}\right)^{4}+7\left(X_{i}^{\prime}-x_{0}\right)^{6}\right]^{T} \\
& X=\left[\Delta k_{0}, \Delta k_{1}, \Delta k_{2}, \Delta x_{0}\right]^{T} \\
& \quad L=x_{i}-\left(x_{i}\right)
\end{aligned}
$$

After iterative computation it got $x_{0}, k_{0}, k_{1}, k_{2}$.

Secondly, compute the focal length $f$.

Carrying on the linearization to this model it can get formula (5) as following,

$$
v_{x_{i}}=\left(x_{i}\right)-x_{i}+\operatorname{tg} a_{i} \cdot \Delta f
$$


With a lot of observation value, formula (5) can be written as a matrix:

$$
\begin{aligned}
& V=A X-L \\
& \text { Where, } A=\operatorname{tga}_{i}, \quad X=\Delta f, L=x_{i}-\left(x_{i}\right)
\end{aligned}
$$

Using the results computed by the first step as the primary data, with iterative computation $f$ can be obtained. Then use this $f$ result as the primary data to compute the parameters of the first step for a second time. Till to all the parameters can get a convergent and stable result, then the final results will have a high precision. The details flow char of this idea is in the Figure6.

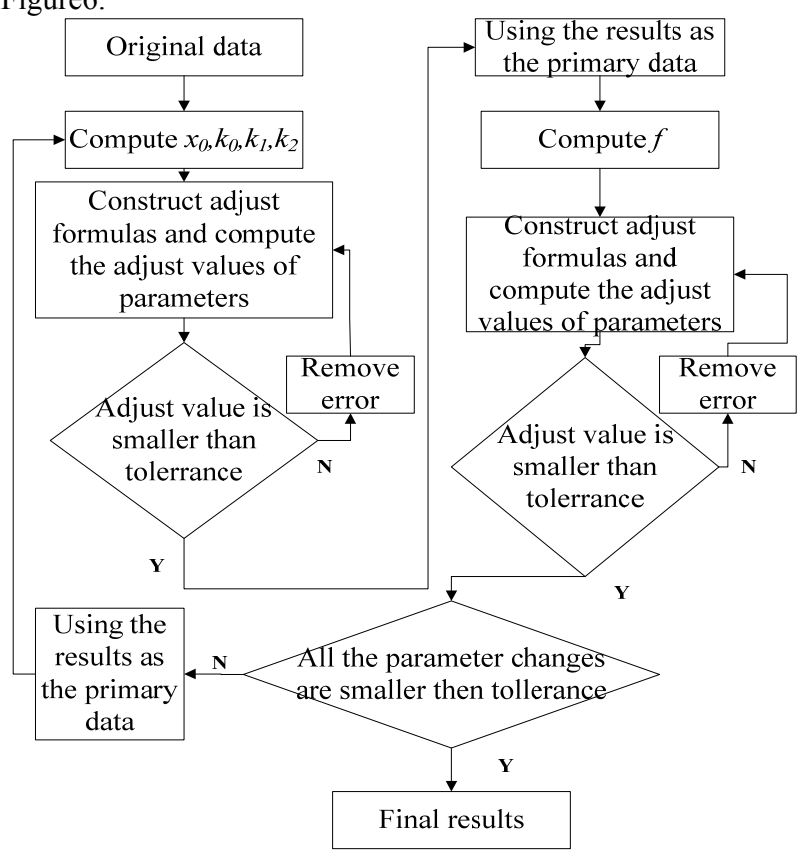

Figure 6 The flow chart of computing parameters

\section{EXPERIMENTS AND THE RESULTS}

Fixed the rigid body onto a lift platform (Figure 5), and then move the platform up and down in certain speed to capture the image and cloud points of the wall of a building. Figure7 is one section image of the experiment wall in our programming, many brick-work joints (characteristic points) on it.

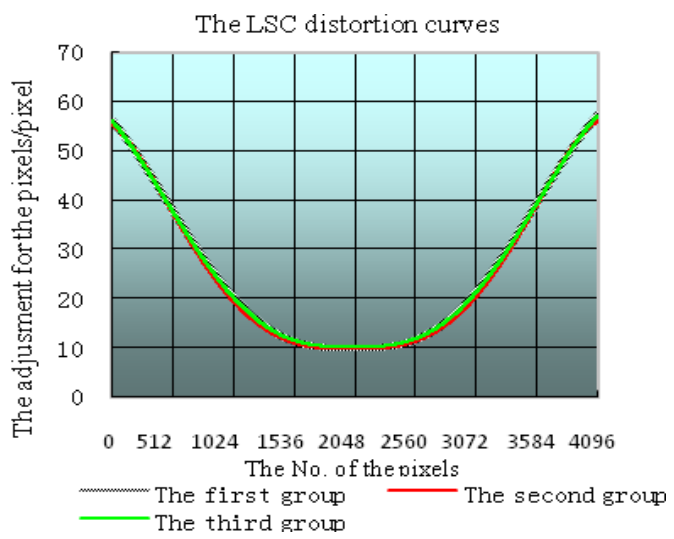

Figure 8 The curves of the distortion

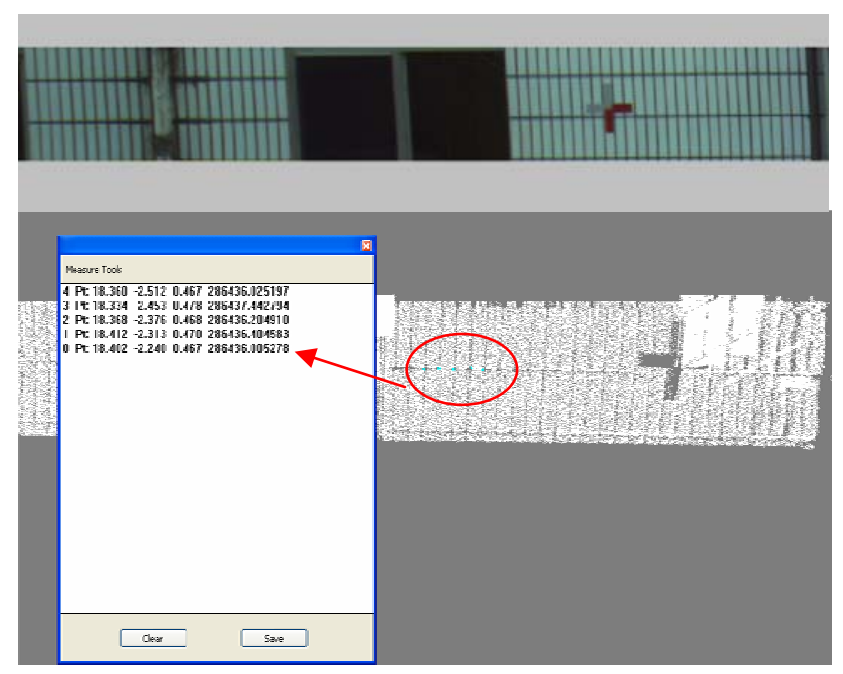

Figure 7 The corresponding of the cloud points and the Image

A programming was written in Visual $\mathrm{C}++$ for computing the calibrate parameters with an error removing algorithm in it.

When

$$
\left|\Delta x_{i}\right|>3 \sqrt{\frac{\sum \Delta x_{i}^{*} \Delta x_{i}}{n-1}}
$$

this pair of data would be regarded as wrong data and would be removed from the original data. Then the other data is used to the iterative computation. Three groups of original data were observed for this experiment, using them to compute the calibrate parameters respectively. Then use the results to calibrate the camera and get the corresponding distortion curve, which were draw in the Figure8 following.

Comparing these three curves, they have similar size and shape (Figure 8), so this calibrate model is right for calibrating the LSC. Meanwhile the results have high precision.

The results of the three group data are in table 1. It also computed the root mean square errors respectively.

$$
\begin{aligned}
& m_{x i 1}= \pm \sqrt{v v /(n-1)}= \pm 0.48 \\
& m_{x i 2}= \pm \sqrt{v v /(n-1)}= \pm 0.42 \\
& m_{x i 3}= \pm \sqrt{v v /(n-1)}= \pm 0.44
\end{aligned}
$$

The pixel size of TVI line scan camera is $10 \mu \mathrm{m}$. Each group original data has more than 150 pairs of data and the maximize root mean square error is $0.51 \times 10=7.14 \mu \mathrm{m}$, which has enough precision to be fused with other data and provide data to City Virtual Reality and Visualization.

Analyzing these three results, it got the root mean square error of the principle point and the focus length as following.

$$
\begin{aligned}
& m_{x 0}= \pm \sqrt{v v /(n-1)}= \pm 0.94 \\
& m_{\mathrm{f}}= \pm \sqrt{v v /(n-1)}= \pm 0.44
\end{aligned}
$$

All the above root mean square error (RMSE) unit is one pixel size $(10 \mu \mathrm{m})$, that is to say the root mean square error of principle point is $0.94 \times 10=9.4 \mu \mathrm{m}$, the focal length one is $0.44 \times 10=4.4 \mu \mathrm{m}$. 


\begin{tabular}{|c|c|c|c|c|c|c|}
\hline groups & $x_{0}$ & $k_{0}$ & $k_{1}$ & $k_{2}$ & $f /$ pixel & RMSE/pixel \\
\hline 1 & 18.93 & $1.71 \times 10^{-8}$ & $-1.30 \times 10^{-14}$ & $2.35 \times 10^{-21}$ & 2482.004 & \pm 0.48 \\
\hline 2 & 18.37 & $1.55 \times 10^{-8}$ & $-1.06 \times 10^{-14}$ & $2.86 \times 10^{-21}$ & 2481.767 & \pm 0.42 \\
\hline 3 & 18.72 & $1.52 \times 10^{-8}$ & $-1.57 \times 10^{-14}$ & $2.76 \times 10^{-21}$ & 2481.60 & \pm 0.44 \\
\hline
\end{tabular}

Table 1 The results and the accurate of the three group data

\section{CONCLUSIONS}

Analyzing the results, this novel approach has high calibration precision. The lens distortion root mean square error is reach to $4.8 \mu \mathrm{m}$, the principal point one is $9.4 \mu \mathrm{m}$ and the focal length one is $4.4 \mu \mathrm{m}$. After calibrate by this approach the image data has totally enough precision to fuse with the laser cloud points. The high precision and stability of this approach makes it can provide reference to calibrate the similar line sensors. In order to improve the precision in the future, the distance between the LSC can the objects could be farther. Decreasing the influence of the laser scanner angle error and the machine error would also be better for improving precision.

\section{ACKNOWLEDGMENTS}

The authors would like to thank Professor Xianlin Liu, 2012 Beijing post-doctoral research activity funding, China National High-Tech Research and Development Program funding (863 Program: 20101AA22202).

\section{REFERENCES}

WEI Bo, ZHANG Aiwu, 2009. Design and Implementation of A Vehicle-borne System of 3D Data Acquisition and Processing. Chinese Journal Of Stereology And Image Analysis, 13, pp.78-86.

CHEN Yunfang, YE Zetian, 2006. Research on Mobile Data Collection System Based on Multi-sensor. Transducer and Micro-system Technologies, 25(12), pp. 23-25.

XIE Wenhan, ZHANG Zuxun, 2004. Camera Calibration Based on Vanishing Points of Multi Image. Acta Geodaetica et Cartographica Sinica, 33(4), pp. 335-340.

FENG Wenhao, 2004. Close Range Photogrammetry. Wuhan: Wuhan Universe Press, pp.185-193.

LIN Zongjian, CUI Hongxia, 2005. Research on the Digital Camera Distortion Calibration. Geomatics and Information Science of Wuhan University, 30(2), pp. 122-125.

Wang Dong, FENG Wenhao, LU Xiushan, 2007. Camera Calibration Of Nikon D1X. Science of Surveying and Mapping, 32(2), pp.33-34.

Radu Horaud, Roger Mohr, and Boguslaw Lorecki, 1993. On Single-Scan line Camera Calibration. IEEE TRANSACTIONS ON ROBOBTICS AND AUTOMATION, 9(1), pp. 71-74.

QU Wenqian, YI Yi, HU Xiaole, 2008. The Application of Single Scan line Camera in Vehicle Born 3D Information Data Acquisition System. Journal of Capital Normal University ( Natural Science Edition), 29(5), pp. 9-11.

ZHANGHongtao, DUAN Fajie, 2007. Study on Calibration of Linear CCD Based on Two Steps. Acta Metrologica Sinica,28(4), pp.311-313.
WANG Liuzhao, 2006. Small Digital and Aerial Photog -rammetry System. KunMing University of Science and Technology, pp. 5-6.

Wang Liuzhao, Qu Laichao, 2009. The Calibration of RA-360 Laser Scanner. The 6th international symposium on digital earth.99.254-256 